\title{
Colorimetric Urinalysis for On-site Detection of Metabolic Biomarkers
}

Sanjida Yeasmin ${ }^{\mathrm{ab \#}}$, Gopal Ammanath ${ }^{\mathrm{ab \#}}$, Yusuf Alic ${ }^{\mathrm{c}}$, Bernhard O. Boehm ${ }^{\mathrm{c}}$, Umit Hakan Yildiz $^{\mathrm{d}}$, Alagappan Palaniappan ${ }^{\mathrm{ab} *}$, Bo Liedberg ${ }^{\mathrm{ab}^{*}}$

${ }^{a}$ Centre for Biomimetic Sensor Science, Nanyang Technological University, Singapore 637553

${ }^{\mathrm{b}}$ School of Materials Science and Engineering, Nanyang Technological University, Singapore 639798

${ }^{c}$ Lee Kong Chian School of Medicine, Nanyang Technological University, Singapore 308232

${ }^{\mathrm{d}}$ Department of Chemistry, Izmir Institute of Technology, Urla, 35430 Izmir, Turkey

*corresponding authors: alps@ntu.edu.sg; bliedberg@ntu.edu.sg

\#Author contributed equally to this work 


\section{Assay protocol for 8-OHdG}

The sensing strategy is based on the monitoring the changes in optical properties of polythiophene (PT) with 8-OHdG aptamer (Apt-2) in the presence and absence of 8-OHdG spiked in urine sample. Complexation of PT and Apt-2 produces a duplex because of the electrostatic interactions between PT and the Apt-2. These interactions induce fluorescence quenching, resulting in a color transition from yellow (PT) to orange (PT-Apt-2). However, the color transition of PT is not observed in the presence of 8-OHdG, as complexation between PT and Apt-2 is perturbed, yielding fluorescent PT-Apt-2-8-OHdG complex, owing to the affinity between Apt-2 and 8-OHdG (formation of G-quadruplex). Therefore, the yellow color (PTApt-2-8-OHdG) and the significantly different orange color (PT-Apt-2) correspond to the presence and the absence of 8-OHdG, respectively, as shown in Fig. S1.

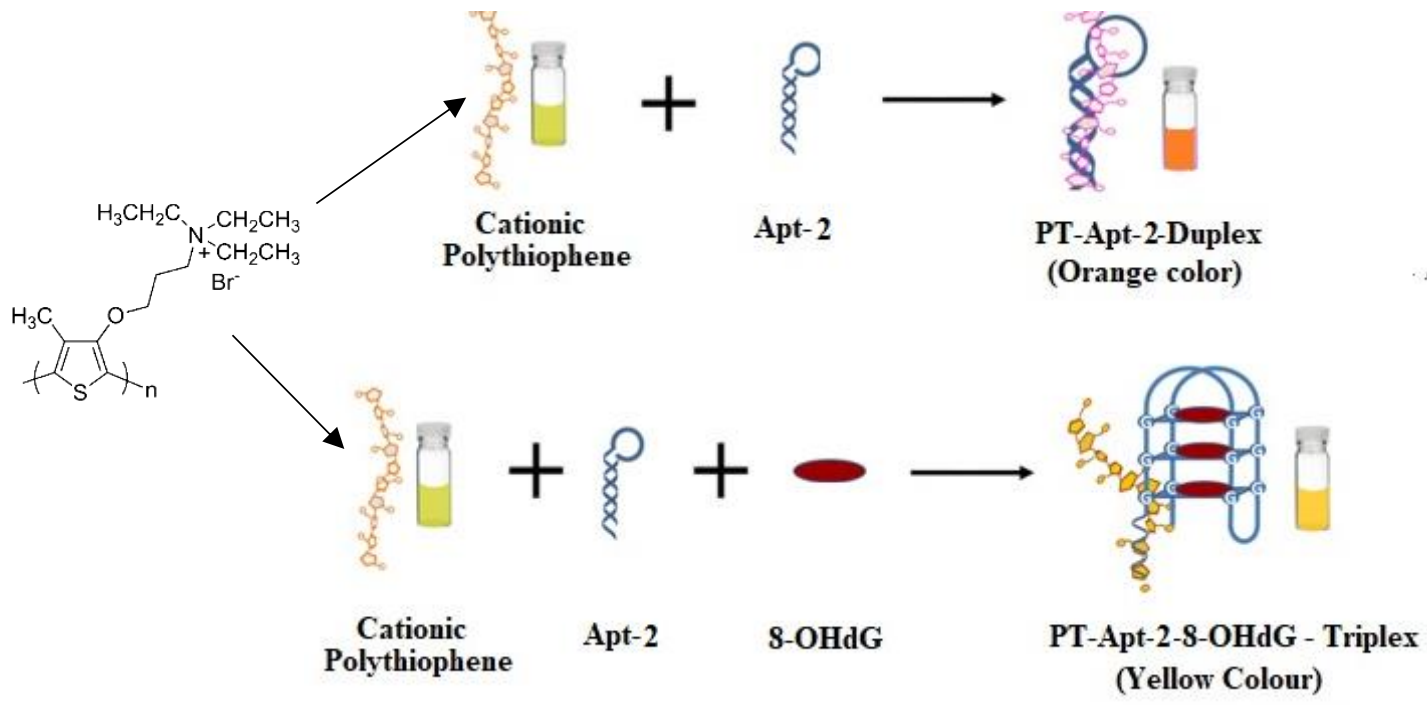

Fig. S1. Schematic illustration for detection of 8-OHdG using PT: Poly(3-(4-methyl-3'thienyloxy)propyltriethylammonium bromide)

\section{Assay protocol for HBV-DNA}

The assay protocol for HBV-DNA is different from that of AGE/ 8-OHdG and follows a typical duplex and triplex interaction scheme between PT-HBV-DNA and PT-PNA-HBVDNA. The sensing strategy is based on monitoring the changes in optical properties of PT-T20, in the absence and in the presence of PNA complementary to HBV-DNA upon complexation with HBV-DNA spiked in urine samples as shown in Fig. S2. PT-T20-HBV-DNA in 10\% urine forms a duplex in the absence of PNA, leading to a color change from yellow to purple. The addition of complementary PNA to HBV-DNA spiked urine samples leads to hybridization 
between PNA and HBV-DNA. Subsequent addition of PT-T20 to the hybridized PNA-HBVDNA results in triplex formation (PT-T20-PNA-HBV-DNA) with insignificant change in color of PT-T20-PNA. Thus, the difference in color between the two vials could be correlated to the concentration of HBV-DNA in urine samples.

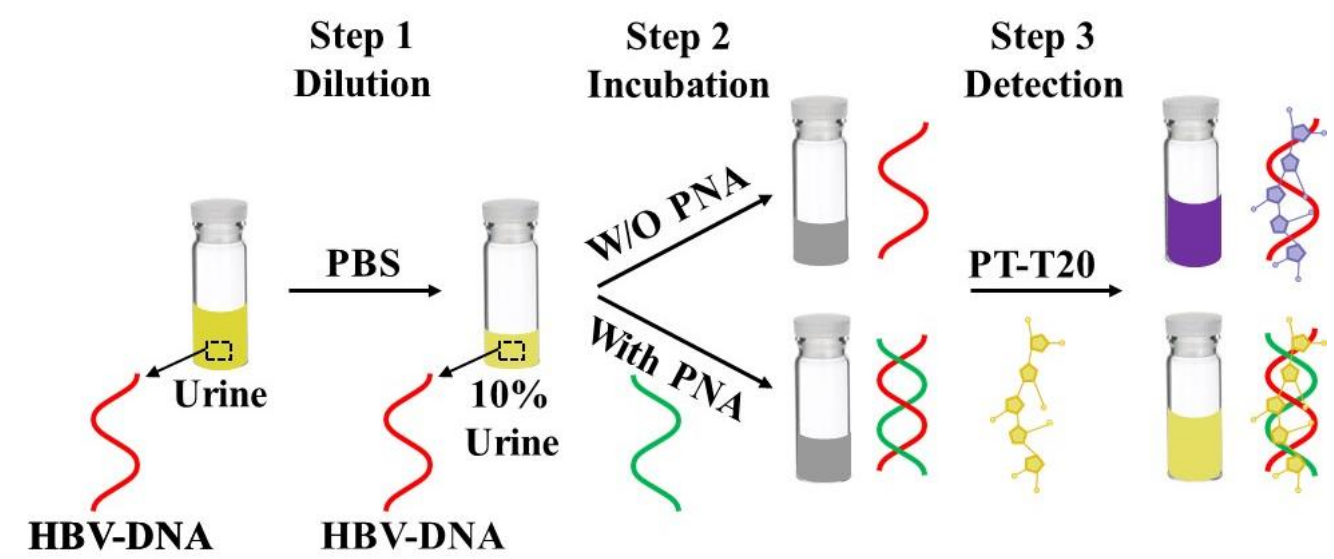

Fig. S2. Schematic illustration of the proposed urinalysis in a homogeneous solution for detection of HBV-DNA

\section{Spectral response of synthetic and urine samples}

The colorimetric and fluorometric responses of synthetic urine, urine samples (three healthy samples and their pooled sample) upon UV excitation are shown in Fig. S3a and Fig. $\mathrm{S} 3 \mathrm{~b}$, respectively.

(a)

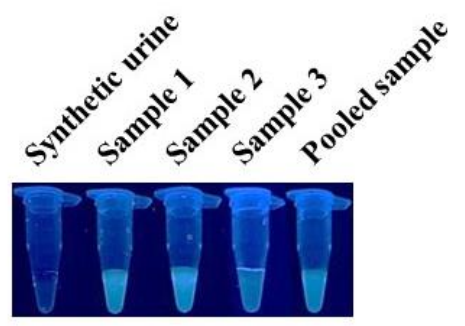

(b)

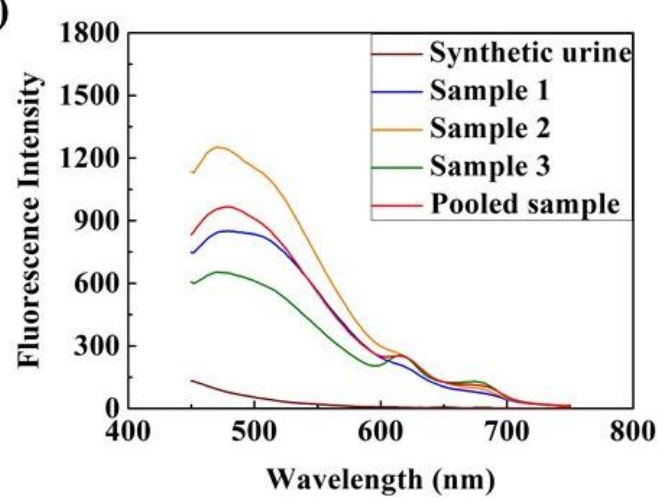

Fig. S3. (a) Colorimetric images of synthetic urine, urine sample 1, urine sample 2, urine sample 3, and pooled sample under UV excitation, and (b) their corresponding fluorescence spectra. 


\section{Effect of surfactants}

The colorimetric and fluorometric responses of PT in the presence and absence of $10 \%$ urine with three different types of surfactants such as T20, TX-100 and SDS at 0.01\% concentration were evaluated as shown in Fig. S4.
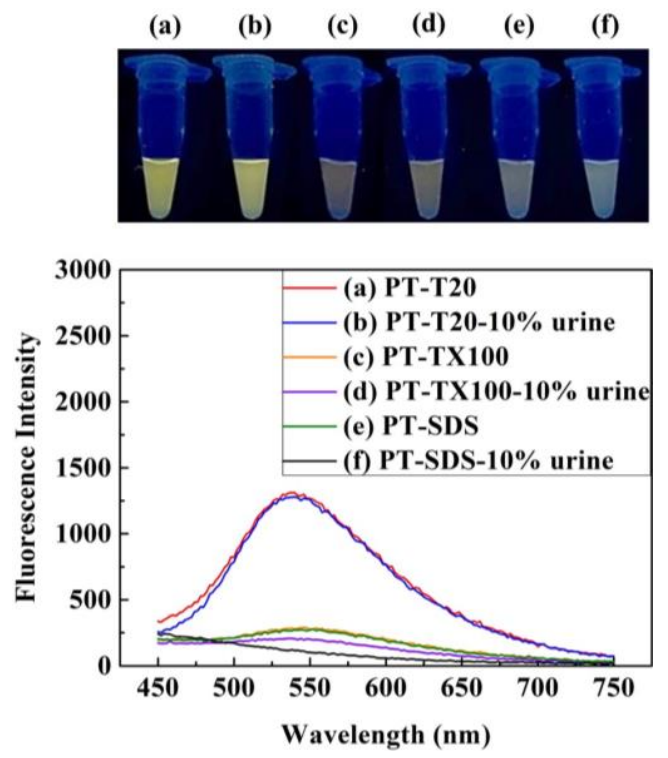

Fig. S4. (a) Colorimetric and fluorescence responses of (a, b) PT-T20, (c, d) PT-TX100, and (e, f) PT-SDS without (a, c, and e) and with (b, d, and f) $10 \%$ urine samples, respectively.

Non-ionic surfactants and other polyethylene glycol (PEG) agents as well as oligosaccharides, like carboxymethylated dextran, have been extensively used in immune-, enzyme-, DNA-assays to suppress non-specific binding (NSB) of matrix components. In many cases the NSB agents are attached to the transducer substrate or electrode or by adding them to the suspension in case of homogenous assay to block it from interacting non-specifically with matrix molecules ${ }^{1-4}$. The attachment to substrate (Glass, Silicon/Silicon Dioxide, ITO, Polymer, Metal) can be covalent, electrostatic or via weaker bonds.

The exact molecular mechanism behind the protective role of the T20, PEG, dextran etc. is still unknown. Apart from the excluded volume (entropic) effect introduced a long time ago for PEG coatings to primarily reduce non-specific protein adsorption, detailed studies on the mechanisms have not been performed ${ }^{5,6}$.

\section{Image processing and data analysis}

Digital images recorded using a mobile phone camera (Samsung S7) were imported to a computer in jpeg format. 75 x 75 pixels of each vials were analyzed using ImageJ software to obtain the color-coded array as well as RGB values. The obtained RGB values were then 
used for quantifying the perceived color changes through $\Delta E$ values using colormine.org as shown in Fig. S5.

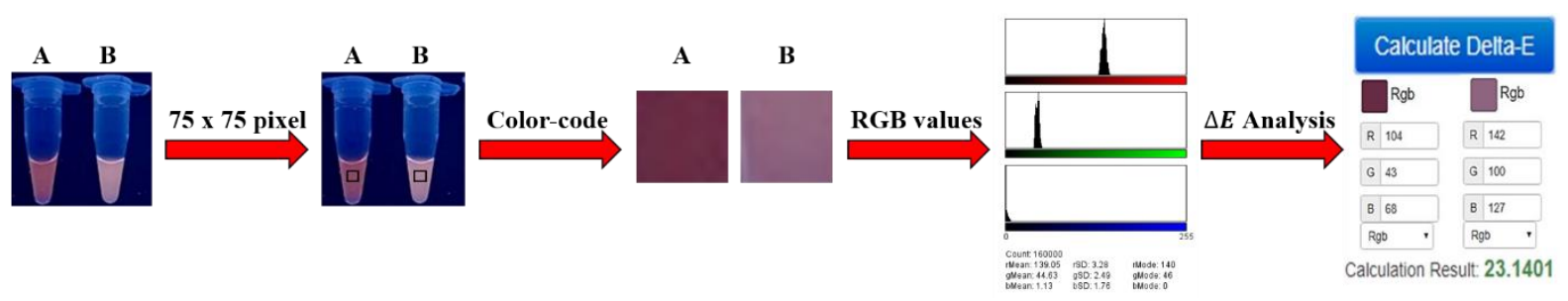

Fig. S5. Major steps in obtaining the color-coded array from vials followed by determining RGB values through ImageJ and calculation of $\Delta E$ values displayed in Fig. 3a (vial A, and B).

\section{LODs from $\Delta E$ values}

The correlation between the $\Delta E$ values and the different biomarker concentrations represented in a semi-logarithmic scale is shown in Fig. S6.
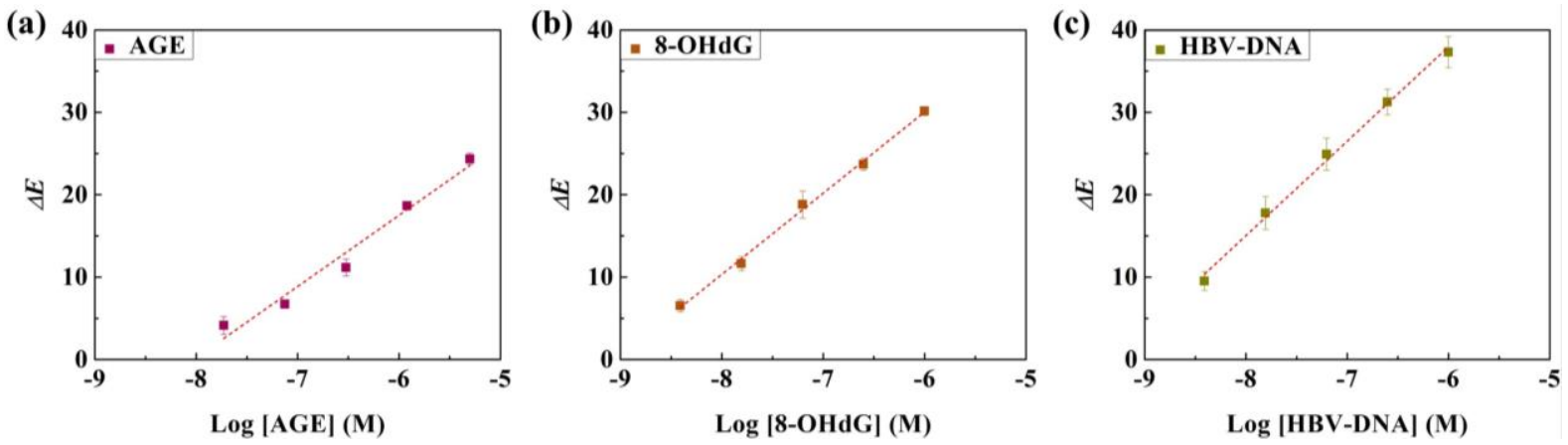

Fig. S6. Semi-logarithmic plots of $\Delta E$ values versus varying concentrations of (a) AGE, (b) 8-OHdG, and (c) HBV-DNA for $(n=3)$.

\section{Control for HBV-DNA assay}

The insignificant color change between PT-T20-PNA-10\% urine and PT-T20-PNAHBV-DNA-10\% urine due to the triplex formation. The concentration of PT-T20 is $75 \mu \mathrm{M}$.

\section{A B}

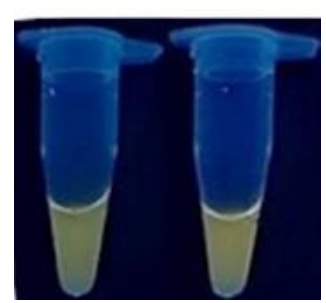

Fig. S7. Colorimetric response of PT-T20-PNA-10\% urine (vial A) and PT-T20-PNA-HBV-DNA (vial B). 


\section{Influence of haemoglobin on assay response}

Fluorescence response of PT-T20-Apt 1 upon addition of $1 \mathrm{mg} / \mathrm{ml}$ haemoglobin $(\mathrm{Hb})$ is shown in Fig. S8. No recovery in fluorescence of PT-T20-Apt1 was observed upon addition of $\mathrm{Hb}$, indicating that $\mathrm{Hb}$ does not interfere with the biomarker assay responses.

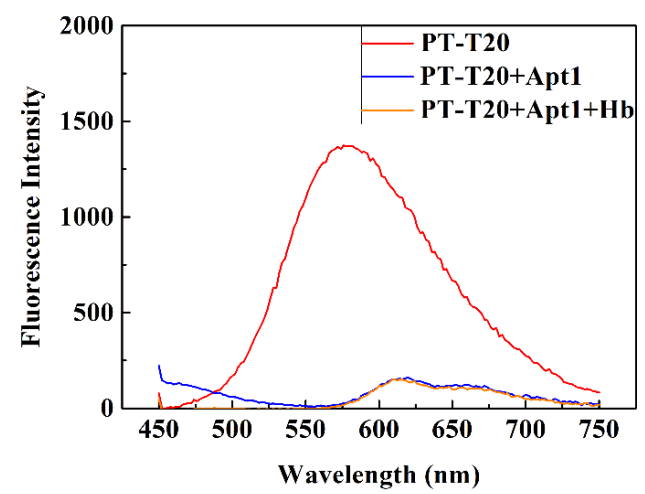

Fig. S8. Fluorescence spectra of PT-T20-Apt1 upon addition of haemoglobin

\section{Generating reference color-coded array}

The mean RGB values of healthy urine samples obtained from three volunteers upon interaction with PT-T20, PT-T20-Apt 1, PT-T20-Apt 2, and PT-T20-PNA, respectively, as shown in Fig. S9 was utilized to create a reference color-coded array. The reference colorcoded array was generated with the help of Microsoft PowerPoint by using the obtained RGB values.

(a)

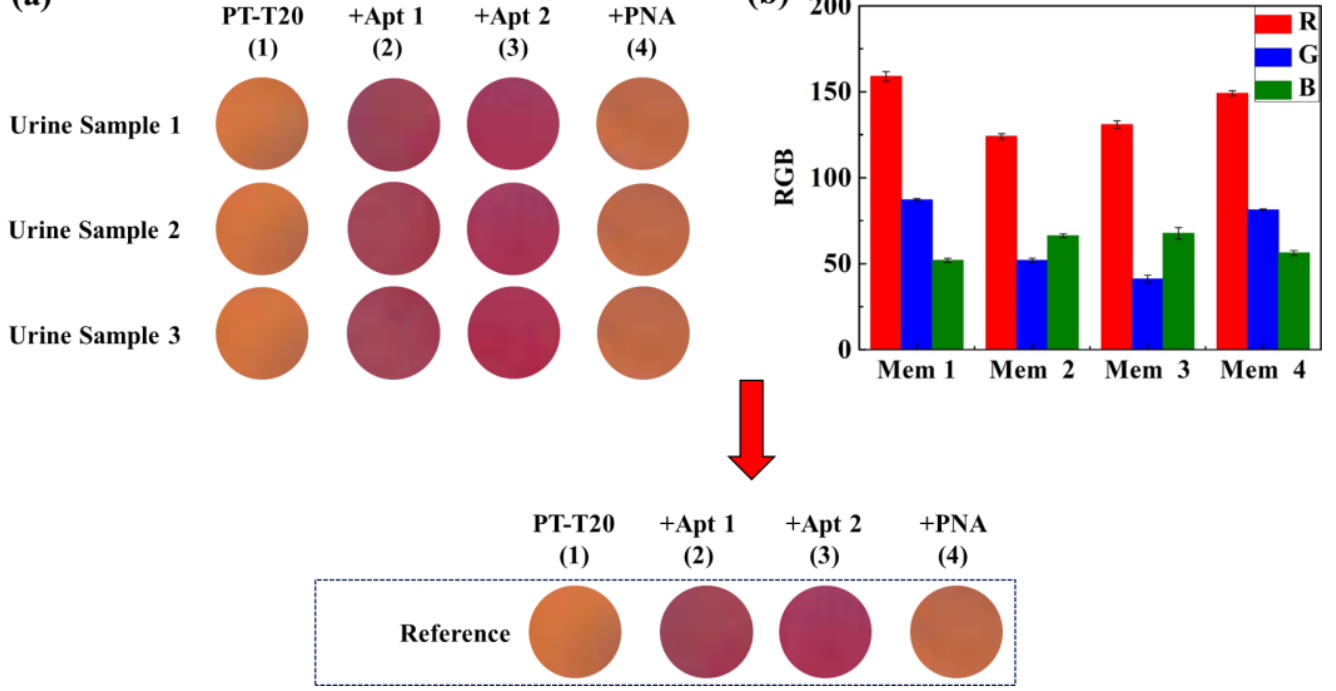

Fig. S9. Procedure for generating reference color-coded array. (a) The colorimetric responses of four individual PVDF membranes numbered as membrane 1, 2, 3 and 4 for healthy urine samples obtained from three volunteers upon interaction with PT-T20, PT-T20-Apt 1, PT-T20-Apt 2, and PT-T20-PNA, respectively and (b) the mean RGB values and standard deviation for $(n=3)$. From the mean values, the array is generated as a reference colorcode for users. 


\section{Influence of $\mathrm{pH}$ and salt concentrations on PT-Apt/DNA spectral responses}

Figure S10 illustrates the influence of $\mathrm{pH}$ and salt concentrations on the interactions between test PT $(0.001 \mathrm{mg} / \mathrm{mL})$ and aptamer $(50 \mathrm{nM}, 29$ mer-DNA, similar in terms of length to the Apt utilized in this study). As observed from Fig. S10, the absorption (a) and fluorescence (b) spectra almost overlaps with each other for varying $\mathrm{pH}$ and salt concentration ranges, illustrating that the electrostatic interactions between PT and DNA are not significantly influenced by $\mathrm{pH}$ or salt concentrations. It should be noted that the protocol in the proposed assay utilizes $10 \%$ urine, diluted in PBS, which is again further diluted by Apt and PT solutions. Therefore, the variations in $\mathrm{pH}$ and salt in urine sample are not expected to significantly influence the colorimetric and fluorometric responses.

a)

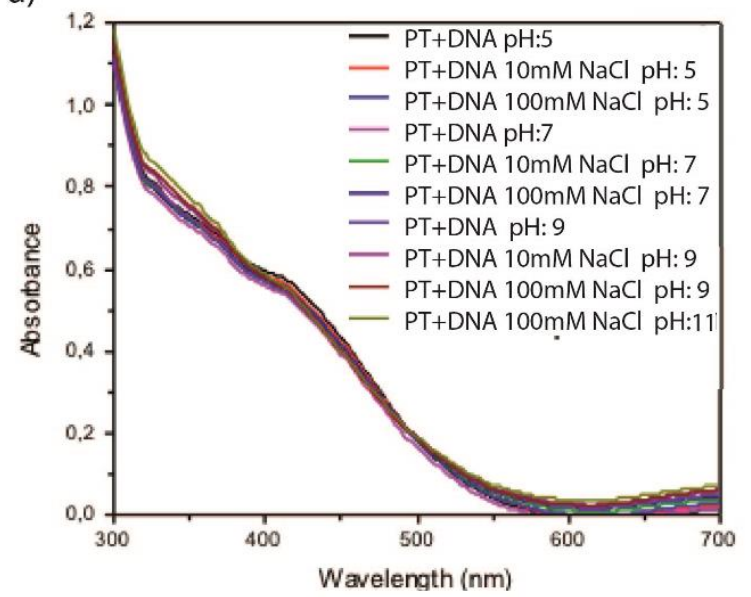

b)

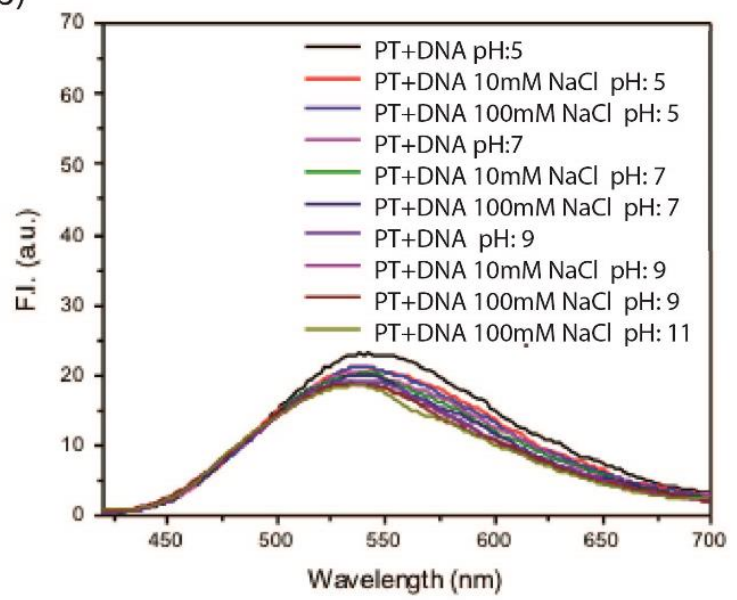

Fig. S10. (a) Absorption and (b) fluorescence responses showing the influence of $\mathrm{pH}$ and salt concentrations on PT-DNA complex

\section{References}

[1] Zhao, W.; Ali, M. M.; Aguirre, S. D.; Brook, M. A.; Li, Y. Paper based Bioassays using Gold Nanoparticle Colorimetric Probes Analytical Chemistry 2008, 80(22), 84318437.

[2] Palumbo, A. J.; Koivunen, M.; Tjeerdema, R. S. Optimization and Validation of a California Halibut Environmental Estrogen Bioassay using a Heterologous ELISA. Science of the Total Environment 2009, 407(2), 953-961.

[3] Sakai, Y.; Hayatsu, M.; Hayano, K. (2002). Use of Tween 20 as a Substrate for Assay of Lipase Activity in Soils. Soil Science and Plant Nutrition 2002, 48(5), 729-734. 
[4] Kenna, J. G.; Major, G. N.; Williams, R. S. Methods for Reducing Non-Specific Antibody Binding in Enzyme-Linked Immunosorbent Assays. Journal of Immunological Methods 1985, 85(2), 409-419.

[5] Jeon, S. I.; Lee, J. H.; Andrade, J. D.; De Gennes, P. Protein-Surface Interactions in the Presence of Polyethylene Oxide: I. Simplified Theory. Journal of Colloid and Interface Science 1991, 142(1), 149-158.

[6] Park, K.; Mao, F. W.; Park, H. The Minimum Surface Fibrinogen Concentration Necessary for Platelet Activation on Dimethyldichlorosilane-Coated Glass. Journal of biomedical materials research 1991, 25(3), 407-420. 